\title{
An Immersive Virtual Reality Curriculum for Pediatric Providers on Shared Decision Making for Hydroxyurea
}

Francis J. Real, MD, MEd, Bradley Cruse, MFA, David Davis MFA, Melissa Klein, MD, MEd, Emily McTate, PhD, Anna Hood, PhD, William Brinkman, MD, MEd, Rogelle Hackworth, BS, Charles Quinn, MD, , Lori Crosby, PsyD

Background: Although hydroxyurea (HU) is an effective disease modifying treatment for sickle cell disease (SCD), uptake remains low in pediatric populations in part due to parental concerns such as side-effects and safety. NHLBI Guidelines recommend shared decision making for HU initiation to elicit family preferences and values; however, clinicians lack specific training. A HU shared decision-making (H-SDM) toolkit was developed to facilitate such discussions (NCT03442114). It includes: 1) decision aids to support parents (brochure, booklet, video narratives, and an in-visit issue card [featuring issues parents reported as key to decision-making about HU]); 2) quality improvement tools to monitor shared decision-making performance; and 3) a curriculum to train clinicians in advanced communication skills to engage parents in shared decision-making. This abstract describes the development and preliminary evaluation of the virtual reality (VR) component of the clinician curriculum.

Objectives: The goals are to: 1) describe the development of a VR simulation for training clinicians in advanced communication skills, and 2) present preliminary data about its tolerability, acceptability, and impact.

Methods: Immersive VR simulations administered via a VR headset were created. The VR environment was designed to replicate a patient room, and graphical character representatives (avatars) of parents and patients were designed based on common demographics of patients with SCD (Figure 1). During simulations, the provider verbally counseled the avatars around HU initiation with avatars' verbal and non-verbal responses matched appropriately. The H-SDM invisit issue card was incorporated into the virtual environment to reinforce practice with this tool. The VR curriculum was piloted for initial acceptability with parents of a child with SCD and clinicians at a children's hospital. Evaluation: Hematology providers participated in the workshop training that included information on facilitating shared decision-making with subsequent deliberate practice of skills through VR simulations. Each provider completed at least one VR simulation. The view through the VR headset was displayed on to a projector screen so others could view the virtual interaction. Debriefing occurred regarding use of communication skills and utilization of the issue card. To assess tolerability, providers reported side effects related to participation. To assess acceptability, providers completed a modified version of the Spatial Presence Questionnaire and described their experience. Impact was assessed by selfreport on a retrospective pre-post survey of confidence in specific communication skills using a 5-point scale (from not confident at all to very confident). Differences in confidence were assessed using Wilcoxon Signed-ranks tests.

Results: Nine providers (5 pediatric hematologists and 4 nurse practitioners at 3 children's hospitals) participated. Tolerability: The VR experience was well tolerated with most providers reporting no side effects (Table 1). Acceptability: All providers agreed or strongly agreed that the VR experience captured their senses and that they felt physically present in the VR 
environment. Providers described the experience as "enjoyable", "immersive", and "fun". One provider noted, "It (the VR simulation) put me in clinic to experience what it felt like to discuss HU and use the tool." Impact: Providers' self-reported confidence significantly improved after VR simulations on 4 of 5 communication skills: confirming understanding, $Z=1.98, p=.05, r=$ .44 , eliciting parent concerns/values, $Z=2.22, p=.03, r=.50$, using an elicit-provide-elicit approach, $Z=1.8, p=.02, r=.50$, minimizing medical jargon, $Z=1.8, p=.07, r=.40$, and using open-ended questions, $Z=1.98, p=.05, r=.44$. Median scores changed by one-point for all responses and effects were medium to large (see Figure 2).

Discussion: The VR curriculum was rated as immersive, realistic, and well-tolerated. Providers endorsed it as a desirable training method. Self-report of confidence in shared decision makingrelated communication skills improved following completion of VR simulation. Thus, initial data support that VR may be an effective method for educating providers to engage parents in shared decision making for $\mathrm{HU}$.

Figure 1: The clinical environment viewed through the virtual reality headset.

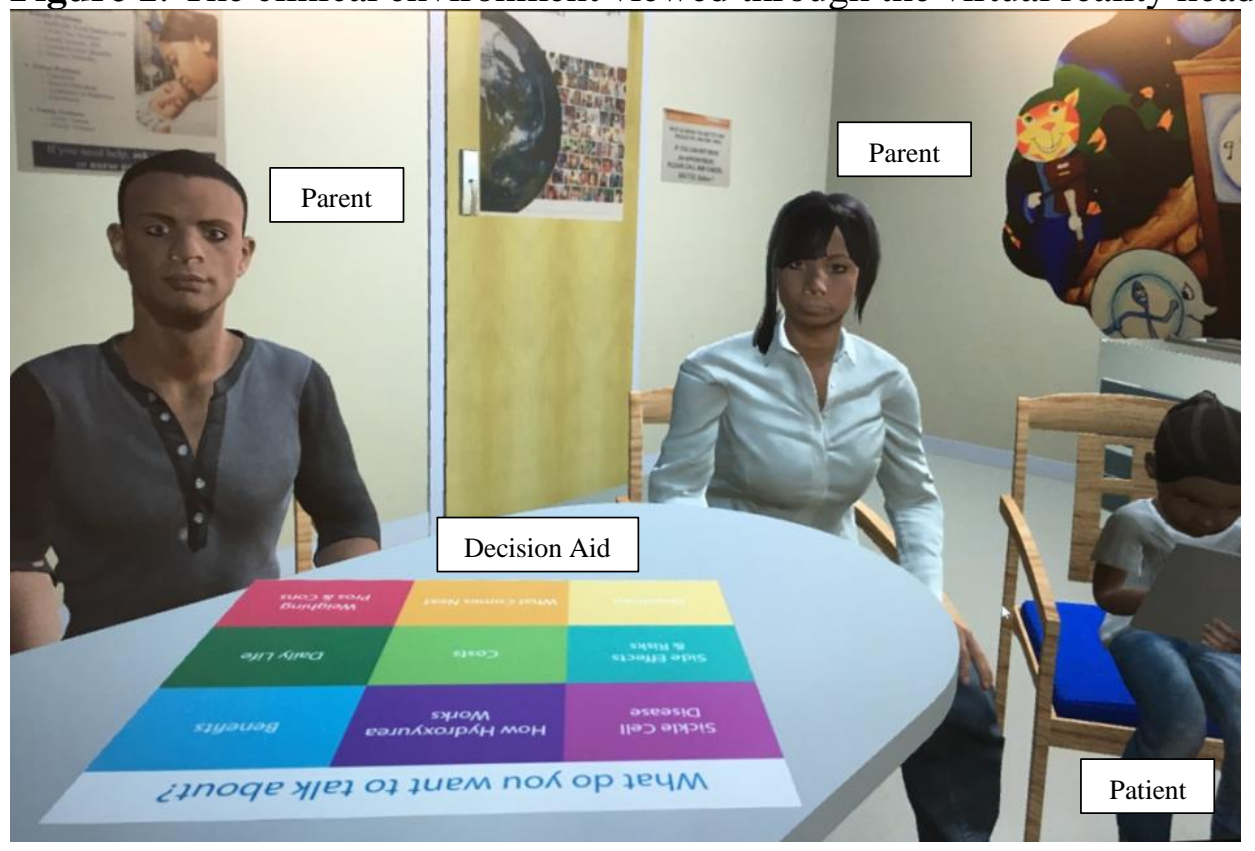

Table 1. Side effects reported by participants following participation in the VR curriculum.

\begin{tabular}{ll}
\hline \multicolumn{1}{c}{ Side effect } & \multicolumn{1}{c}{$\begin{array}{c}\text { Providers } \\
\mathbf{N}(\%)\end{array}$} \\
\hline Blurred vision & $1(11.1 \%)$ \\
Burping & $0(0 \%)$ \\
Difficulty concentrating & $0(0 \%)$ \\
Disorientation & $1(11.1 \%)$ \\
Dizziness & $1(11.1 \%)$ \\
Drowsiness & $0(0 \%)$ \\
Eye strain & $1(11.1 \%)$
\end{tabular}




\begin{tabular}{ll} 
Fatigue & $0(0 \%)$ \\
General Discomfort & $0(0 \%)$ \\
Headache & $0(0 \%)$ \\
Nausea & $0(0 \%)$ \\
Salivation & $0(0 \%)$ \\
Stomach awareness & $0(0 \%)$ \\
Sweating & $0(0 \%)$ \\
\hline
\end{tabular}

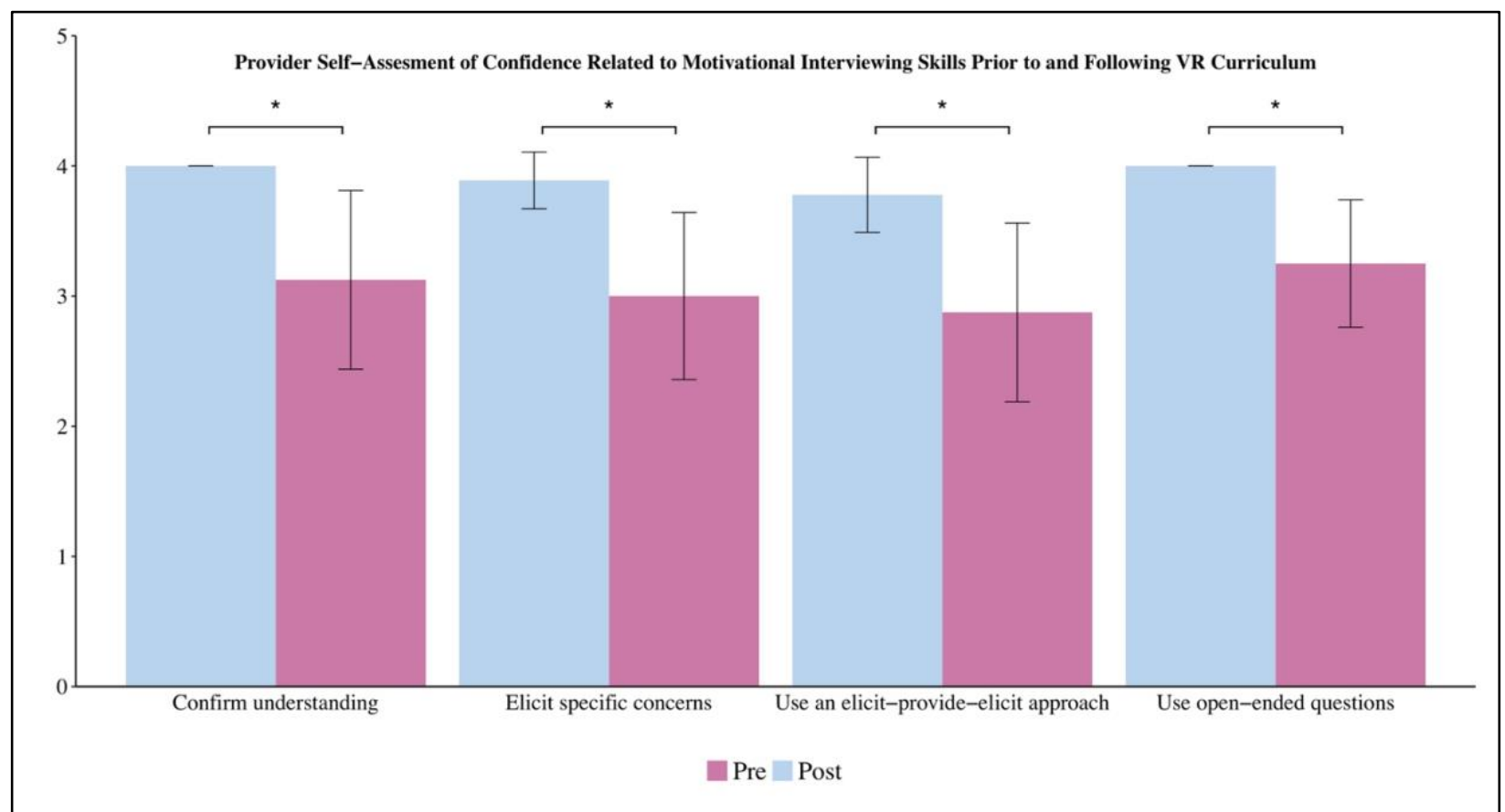

Figure 2: Results of a provider retrospective pre-post survey regarding confidence on a 5-point scale (from not confident at all to very confident) performing motivational interviewing skills. $*$ $=p<.05$. 